\title{
RUNX 3, apoptosis 0: a new gastric tumour suppressor
}

\section{S F Moss}

There is convincing evidence that Runx 3 is a new and important tumour suppressor in gastric cancer

$n$
th
et comparison with our knowledge of the detailed sequence of somatic genetic changes occurring during colon carcinogenesis, relatively few tumour suppressors or oncogenes have been commonly implicated during the multistage progression to cancer in the stomach. Now, in a collaborative venture reminiscent of their countries' successful co-management of the 2002 World Cup $\mathrm{Li}$ and 26 colleagues from South Korea and Japan have convincingly identified Runx 3 as a new and important tumour suppressor in gastric cancer.

Runx 3 is one of three mammalian homologues of Runt, a gene important for segmentation in Drosophila. In general, Runx gene products function as transcriptional regulators of the Smad gene family that in turn mediate the downstream effects of signalling by transforming growth factor $\beta$ (TGF- $\beta$ ) and its homologues. ${ }^{1}$ Runx $\mathrm{l}$ is critical for mammalian haematopoiesis and Runx 2 for bone formation; Runx 1 has been implicated in the aetiology of leukaemia and Runx 2 in lymphoma. What does Runx 3 do? In an exhaustive and thorough series of critical experiments, Li et al report that Runx 3 is strongly expressed in the gastrointestinal tract of mice. To determine its function here, mice deficient in Runx 3 were generated. These animals died soon after birth (probably due to hypoglycaemia) but they also were noted to have gastric mucosal hyperplasia. Histological and functional studies in the Runx 3 knockout mice indicated that the observed gastric mucosal hyperplasia was due not to increased gastric epithelial proliferation but to insufficient "physiological" cell loss through apoptosis. Furthermore, isolated gastric epithelial cells from the Runx $3^{--}$mice were particularly insensitive to apoptosis that is normally stimulated by TGF- $\beta$. This function of Runx 3 as an apoptosis inducer is consistent with its selective localisation at the luminal end of the gastric gland (where apoptosis occurs most frequently) and its absence from the gastric proliferative zone.

As failure to appropriately activate apoptotic cell death is a feature of cancer cells, Li et al then investigated Runx 3 in human gastric cancer. They reported that the majority of cell lines and primary

Li QL, Ito K, Sakakura C, et al. Causal relationship between the loss of RUNX3 expression and gastric cancer. Cell 2002;109:113-24.

Runx3/Pebp2alphaC null mouse gastric mucosa exhibits hyperplasias due to stimulated proliferation and suppressed apoptosis in epithelial cells, and the cells are resistant to growth-inhibitory and apoptosisinducing action of TGF-beta, indicating that Runx3 is a major growth regulator of gastric epithelial cells. Between $45 \%$ and $60 \%$ of human gastric cancer cells do not significantly express RUNX3 due to hemizygous deletion and hypermethylation of the RUNX3 promoter region. Tumorigenicity of human gastric cancer cell lines in nude mice was inversely related to their level of RUNX3 expression, and a mutation (R122C) occurring within the conserved Runt domain abolished the tumorsuppressive effect of RUNX3, suggesting that a lack of RUNX3 function is causally related to the genesis and progression of human gastric cancer.

cancers that were evaluated had absent or low Runx 3 expression due either to hemizygous deletion of the Runx 3 gene or, more commonly, to transcriptional silencing by promoter hypermethylation. The degree of loss of Runx 3 was dependent on the extent of diseaseapproximately $40 \%$ of tumours in stage 1 had no detectable Runx 3 expression compared with about $90 \%$ of stage IV tumours. Intriguingly, Runx 3 expression could be restored in some gastric cancer cell lines by treating cells with $5^{\prime}$-aza- $2^{\prime}$ deoxycytidine or trichostatin, agents that reverse promoter hypermethylation in vitro. Finally, to further support the concept that loss of Runx 3 function is an important contributory cause of gastric malignant transformation (rather than an effect), Li et al demonstrated using standard tumorigenicity assays that restoring Runx 3 function by gene transfection in cells lacking Runx 3 caused marked inhibition of tumour growth. This inhibition was not observed with a Runx 3 construct in which a single nucleotide coding a putative functional region had been mutated, thus providing convincing evidence for Runx 3 as a gastric tumour suppressor gene.

The findings of this report will spur many further investigations. Some obvious questions to be answered initially relate to the timing of loss of Runx 3 expression. This paper describes Runx 3 expression as being lowest in the most advanced cancers, yet Runx 3 was also reduced in some areas of intestinal metaplasia adjacent to the tumour-is Runx 3 also downregulated in intestinal metaplasia in the absence of gastric cancer? Is Runx 3 expression lost in gastric cancers of the diffuse phenotype too? What is the relationship of Runx 3 to the major environmental gastric carcinogen Helicobacter pylori, or to other genes commonly mutated in gastric cancer such as p53 or E-cadherin? It is interesting to note that TGF- $\beta$ and Smad4 knockout mice each display gastric hyperplasia ${ }^{12}$ and that insensitivity to TGF- $\beta$ mediated by several different pathways is frequent in gastric cancer, ${ }^{3}$ emphasising the importance of the TGF- $\beta /$ Run $\times 3 /$ Smad 4 pathway in restraining abnormal epithelial growth in the stomach. As Runx 3 is expressed throughout the luminal gastrointestinal tract, what of its function in the intestines? Is it also reduced in expression in colonic neoplasia?

Apart from the relevance of this work to the pathogenesis of gastric cancer, the paper illustrates several important general principles. Firstly, it demonstrates how very basic research (from Drosophila) can be quickly translated to yield important insights into the aetiology and potentially the prognosis of a common clinical condition. As is becoming an increasingly familiar scenario, the experimental deletion of a gene in mice whose function was obscure led to an unexpected phenotype-in this case that of gastric hyperplasia. Impressive in this study was the ability of the research team to then move rapidly from genetic to anatomical to functional analyses, leading ultimately to very obvious clinical implications. Secondly, here is an 
excellent example where failure to appropriately activate physiological apoptosis contributes to tissue expansion and neoplasia. Thirdly, this paper illustrates the importance of epigenetic changes in the aetiology of disease. Promoter methylation is now commonly recognised as a major determinant of gene expression. The fact that Runx 3 expression could be restored by promoter demethylation in cell culture suggests that strategies to reactivate silenced genes may one day be of therapeutic value clinically.

Gut 2003;52:12-13

\section{Author's affiliation}

S F Moss, Division of Gastroenterology,

Department of Medicine, Rhode Island Hospital,

593 Eddy St, APC 445, Providence, RI 02903

USA; smoss $1 @$ pol.net

\section{REFERENCES}

1 Weinstein $M$, Yang $X$, Deng $C$. Functions of mammalian Smad genes as revealed by targeted gene disruption in mice. Cytokine Growth Factor Rev 2000;1 1:49-58.

2 Crawford SE, Stellmach V, Murphy-Ullrich JE, et al. Thrombospondin-1 is a major activator of TGF-beta 1 in vivo. Cell 1998;93:1 159-70.

3 liichi $\mathbf{H}$, lkenoue T, Kato $\mathbf{N}$, et al. Systematic analysis of the TGF-beta-Smad signalling pathway in gastrointestinal cancer cells. Biochem Biophys Res Commun 2001;289:350-7.

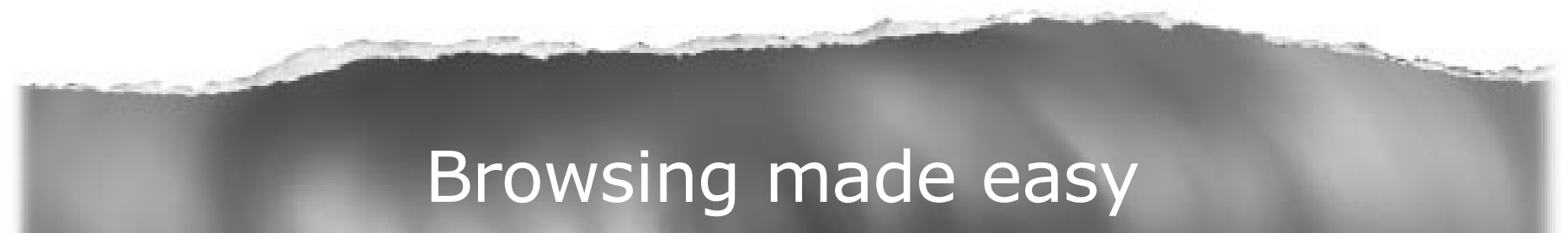

\section{Collections}

With a single click Collections allows you to find all articles that have been published in your chosen subject. Select from over 200 clinical and non-clinical topic collections and/or cross search other specialist journals, the BMJ and Cochrane Reviews

www.gutjnl.com 